\title{
Sub-Microarcsecond Astrometry and New Horizons in Relativistic Gravitational Physics
}

\author{
Sergei M. Kopeikin \\ Department of Physics and Astronomy, 223 Physics Building, \\ University of Missouri-Columbia, Columbia, MO 65211, USA \\ Carl R. Gwinn \\ Department of Physics, Broida Hall, University of California at Santa \\ Barbara, Santa Barbara, CA 93106, USA
}

\begin{abstract}
Attaining the limit of sub-microarcsecond optical resolution will completely revolutionize fundamental astrometry by merging it with relativistic gravitational physics. Beyond the sub-microarcsecond threshold, one will meet in the sky a new population of physical phenomena caused by primordial gravitational waves from the early universe and/or different localized astronomical sources, space-time topological defects, moving gravitational lenses, time variability of gravitational fields of the solar system and binary stars, and many others. Adequate physical interpretation of these yet undetectable sub-microarcsecond phenomena cannot be achieved on the ground of the "standard" post-Newtonian approach (PNA), which is valid only in the near-zone of astronomical objects having a time-dependent gravitational field. We describe a new, post-Minkowskian relativistic approach for modeling astrometric observations having sub-microarcsecond precision and briefly discuss the lightpropagation effects caused by gravitational waves and other phenomena related to time-dependent gravitational fields. The domain of applicability of the PNA in relativistic space astrometry is outlined explicitly.
\end{abstract}

\section{Theoretical principles of relativistic astrometry}

For a long time the basic theoretical principles of general relativistic astrometry in the solar system were based on using the post-Newtonian approximate solution of the Einstein equations (Soffel, 1989; Brumberg, 1991; Will, 1993). The metric tensor of the post-Newtonian solution is an instantaneous function of coordinate time $t$. It depends on the field point, $\mathbf{x}$, and the coordinates, $\mathbf{x}_{a}(t)$, and velocities, $\mathbf{v}_{a}(t)$, of the gravitating bodies, and is valid only inside the near zone of the solar system because it involves expansion of retarded field integrals with respect to the small parameter $v_{a} / c$ (Fock, 1959). This expansion restricts the domain of validity for which the propagation of light rays can be considered from the mathematical point of view in a self-consistent manner by the boundary of the near zone. Finding a solution of the equations of light propagation in the near zone of the solar system, for instance, can be achieved 
by expanding the positions and velocities of the solar system bodies in a Taylor series around some fixed instant of time $t^{*}$, their substitution into the equations of motion of photons, and their subsequent integration with respect to time. Such an approach is theoretically well-justified for a proper description of radar (Reasenberg et al., 1979) and lunar laser ranging (Williams et al., 1996) experiments, and interpretation of the Doppler tracking of satellites. However, this static-in-time approach meets with unsurpassable difficulties if one wants to integrate the equations of light propagation from any object lying beyond the limits of the solar system, where the gravitational field cannot be considered as static if one decides to search for sub-microarcseconds astrometric effects. This is because planets are moving around the Sun and, hence, the gravitational field is changing.

An additional problem arising in expansion about a single instant of time $t^{*}$ is how to determine that fiducial instant of time to which the coordinates and velocities of gravitating bodies should be anchored. The answer is obscured if one works in the framework of the PNA scheme, which disguises the hyperbolic character of the Einstein equations for the gravitational field, and does not allow us to distinguish between advanced and retarded solutions of the field equations (Damour et al., 1991). For this reason, propagation of light rays, which always takes place along the null characteristics of a light cone, differs in the postNewtonian scheme from that of the gravitational field itself, which propagates (in the framework of this scheme) instantaneously with infinite speed. Thus, the true causal relationship between the position of the light particle and the location of the light-deflecting bodies in the system is violated, which leads to a need for artificial assumptions about the initial values of the positions and velocities of the bodies for integration of equations of light propagation. One reasonable choice is to fix the coordinates and velocities of the body at the moment of the closest approach of the light ray. Such an assumption was used by Klioner \& Kopeikin (1992), who proved that it minimizes the magnitude of residual terms of the post-Newtonian solution of the equations of light propagation. Among other difficulties in the application of the PNA to solving the problem of propagation of light rays beyond its domain of applicability is the logarithmic divergence of the post-Newtonian metric tensor at large distances from the solar system. Almost all extra-solar luminous objects visible in the sky lie far beyond the allowed distance and, hence, the results of integration of the equations of light propagation from, e.g., stars in our galaxy or extra-galactic objects performed previously by various authors on the premise of the implementation of the post-Newtonian metric tensor cannot be considered as rigorous and conclusive, because the magnitude of residual terms of such an integration were never discussed.

A real breakthrough in the problem of integration of equations of light propagation in time-dependent gravitational fields has been achieved only recently by Kopeikin et al. (1999), where astrometric and timing effects of the near, intermediate, and far regions of localized gravitational source emitting gravitational waves were precisely calculated and thoroughly discussed on the basis of the post-Minkowskian approximation (PMA) scheme that is free of the drawbacks of the standard PNA. Further progress in solving this problem was made in the paper by Kopeikin \& Schäfer (1999), where propagation of light in the field of arbitrary-moving massive monopole particles was described in detail. All 
possible astrometric effects in VLBI, pulsar timing, Doppler tracking, and so on were considered, including all retardation phenomena in the propagation of a gravitational field from the particles to the photon. We will present a precise description of the gravitomagnetic effects of particles due to their translational and rotational motion in an upcoming publication (Kopeikin \& Mashhoon, 2000). The model we are using now is so flexible and physically meaningful that it can be applied for the treatment of any modern astrometric observation as well as for the prediction of numerous physical effects caused by time-dependent gravitational fields including gravitational waves from the early universe, localized sources of gravitational waves like supernova explosions or binary stars, moving and rotating bodies, oscillations of stars, topological defects, and many others.

\section{Advanced relativistic model of light propagation}

In this section we briefly describe the relativistic model of light propagation used in our calculations (Kopeikin \& Schäfer, 1999; Kopeikin et al., 1999; Kopeikin \& Mashhoon, 2000) and its application for the calculation of some effects that are important in gravitational physics. The relativistic model of light propagation includes three main constituents: (1) equations of the gravitational field, (2) equations of light propagation, and (3) initial and/or boundary conditions. We formulate the equations of gravitational field in a harmonic coordinate system with arbitrary origin in space. For convenience it can be put at the center-ofmass of the source of gravitational field, but this is not essential. We also work in the linear approximation with respect to the universal gravitational constant $G$ without expansion in the velocities of bodies. The metric tensor of the gravitational field can be found immediately from the energy-momentum tensor of the source of the gravitational field. The metric tensor is a function of the retarded time argument $s$ related to the running time $t$ and to the coordinates $\mathbf{x}$ of the photon, as well as the retarded coordinates $\mathbf{x}_{a}(s)$ of the light-deflecting body by the light-cone equation $s=t-\left|\mathbf{x}-\mathbf{x}_{a}(s)\right|$. With the metric tensor in hand we calculate the Christoffel symbols and derive the equations of light propagation, which are second-order ordinary differential equations with retarded argument s. Exact solutions of such equations became available only after we found a series of transformations which brought the equations to the integrable form (Kopeikin \& Schäfer, 1999; Kopeikin et al., 1999).

Assuming that the light is emitted at a point $\mathbf{x}_{0}$ at time $t_{0}$, and that its direction of propagation is given at past null infinity as a unit vector $\mathbf{k}$, we integrate the equations of light propagation with no other restrictions. The result is the trajectory of the light ray perturbed by the time-dependent gravitational field, and written as a function of spatial coordinates of the photon, $\mathbf{x}$, with parametric dependence on the coordinates of the light-deflecting bodies, taken at the retarded time $s$. All predictions of possible relativistic effects (time delay, deflection angle, intensity, polarization angle, etc.) follow directly from the parametric representation of the trajectory. For more details see Kopeikin et al. (1999), Kopeikin \& Schäfer (1999), and Kopeikin \& Mashhoon (2000). 


\section{Sub-microarcsecond astrometry and relativistic gravitational ef- fects}

In this section we briefly describe what kind of predictions one can make beyond the sub-microarcsecond level using the aforementioned advanced integration technique. Sub-microarcsecond space-interferometry optical technology along with the advanced post-Minkowskian theory of light propagation in timedependent gravitational fields, particularly including plane and multipolar gravitational waves, opens outstanding new perspectives for experimental gravitational physics. Here we briefly outline some of the relativistic effects that could be precisely calculated and measured. These effects include:

1. Deflection of light in the higher-order PMA quadratic in $G$, allowing a static-gravitational-field test of alternative theories of gravity with much better precision than in the weak-gravitational-field regime linear in $G$.

2. Deflection of light caused by gravitational waves emitted by binary stars and other periodic and/or non-periodic sources of gravitational waves, which may allow tests of alternative theories of gravity in the radiative gravitational-wave regime.

3. Various effects caused by a pre-assumed hypothetical difference in the speeds of propagation of gravity and electromagnetic waves. This difference may arise, for example, as a result of propagation of light rays through the interstellar medium with the refraction index different from unity or within the framework of an alternative theory of gravity. Difference in these speeds would bring about deviation of the gravitational null cone from the electromagnetic one, which can be tested in observations of the deflection of light by giant planets of the solar system or extra-solar gravitational lenses.

4. Specific pattern of proper motions of quasars over the whole sky caused by primordial gravitational waves from the early universe. Certain progress in solving this problem both theoretically and observationally has been achieved by Pyne et al. (1996) and by Gwinn et al. (1997). However, a step over the sub-microarcsecond threshold would make the research in this direction much more profound.

5. Relativistic effect of secular aberration caused by the circular motion of the solar system with respect to the galactic barycenter. Visualization of the secular aberration effect requires calculation of a light-ray trajectory in the galactic reference frame with the subsequent relativistic space-time transformation from this frame to the proper reference frame of the observer. Some details of calculations are given by Kopeikin (1992) and Klioner \& Kopeikin (1992).

6. Cosmological gravitational lens effects for deflectors with variable parameters such as mass, spin, quadrupole moment, etc. A super-massive binary black hole in an AGN emitting gravitational waves on a cosmological timescale is an example of such a source. 
7. Testing astrometric effects caused by relic space-time topological defects like vibrating cosmic strings, oscillating cosmic loops, gases of monopoles, textures, etc.

8. Study of limitations on the precision of fundamental astrometric reference frames imposed by gravitational lensing events due to flybys of stars in our galaxy (Sazhin et al., 1998), gravitational waves from an ensemble of galactic binary stars (Kopeikin, 1999), and other related phenomena.

We are now critically examining these effects in order to work out an appropriate observational strategy which might be used for their detection by VLBI and/or FAME, SIM, and GAIA space-astrometric missions.

Acknowledgments. We thank Bahram Mashhoon and Mikhail Sazhin for a critical reading of the manuscript and useful comments.

\section{References}

Brumberg, V.A., 1991, Essential Relativistic Celestial Mechanics, Bristol: Adam Hilger.

Fock, V.A., 1959, The Theory of Space, Time and Gravitation, London: Pergamon Press.

Damour, T. \& Schäfer, G., 1991, Phys. Rev. Lett., 66, 2549.

Gwinn, C.R., Eubanks, T.M., Pyne, T., Birkinshaw, M. \& Matsakis, D.N., 1997, Astrophys. J., 485, 87.

Klioner, S.A. \& Kopeikin, S.M., 1992, Astron. J., 104, 897.

Kopeikin, S.M., 1992, Proc. MG6 Meeting on GR, eds. H. Sato, T. Nakamura, and R. Ruffini, Singapore: World Sci. Publ., 1654-56.

Kopeikin, S.M., 1999, Proc. XXXIVth Rencontres de Moriond on "Gravitational Waves and Experimental Gravity," Les Arcs, January 1999 (in press).

Kopeikin, S.M., Schäfer, G., Gwinn, C.R., \& Eubanks, T.M., 1999, Phys. Rev. $D, \mathbf{5 9}, 084023$.

Kopeikin, S. \& Schäfer, G., 1999, Phys. Rev. D, 60, 124002.

Kopeikin, S.M. \& Mashhoon, B., 2000, in preparation.

Pyne, T., Gwinn, C.R., Birkinshaw, M., Eubanks, T.M., \& Matsakis, D.N., 1996, Astrophys. J., 465, 566.

Reasenberg, R.D., Shapiro, I.I., Macneil, P.E., Goldstein, R.B., Breidenthal, J.C., Brenkle, J.P., Cain, D.L., Kaufman, T.M., Komarek, T.A., \& Zygielbaum, A.I., 1979, Astrophys. J., 234, L219.

Sazhin, M.V., Zharov, V. E., Volynkin, A. V., \& Kalinina, T. A., 1998, MNRAS, 300, 287.

Soffel, M.H., 1989, Relativity in Astrometry, Celestial Mechanics and Geodesy, Berlin: Springer-Verlag.

Will, C.M., 1993, Theory and Experiment in Gravitational Physics, Cambridge: Cambridge University Press.

Williams, J.G., Newhall, XX, \& Dickey, J.O., 1996, Phys. Rev. D, 53, 6730. 